\title{
Enhancing Character Posing by a Sketch-Based Interaction
}

Simone Barbieria,b, Nicola Garauc, Wenyu Hud, Zhidong Xiaoa, Xiaosong Yanga

aBournemouth University, ${ }^{b}$ Centre for Digital Entertainment, cUniversity of Cagliari, dGannan Normal University
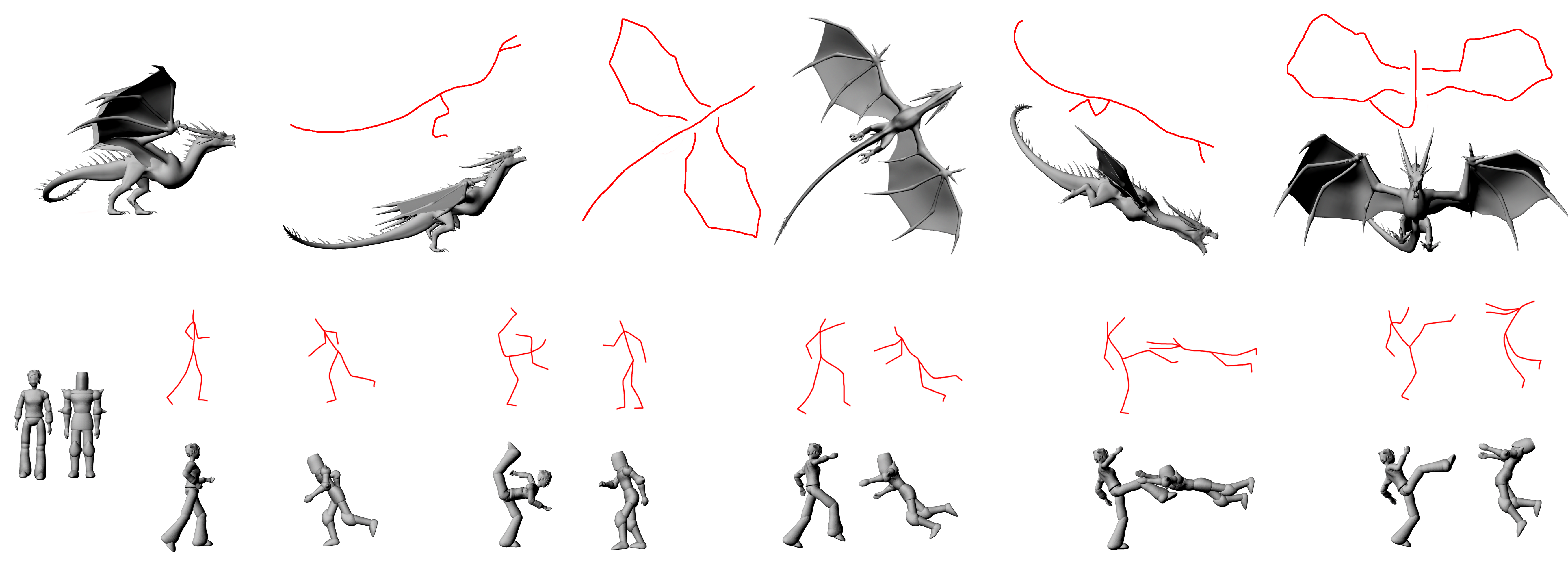

资
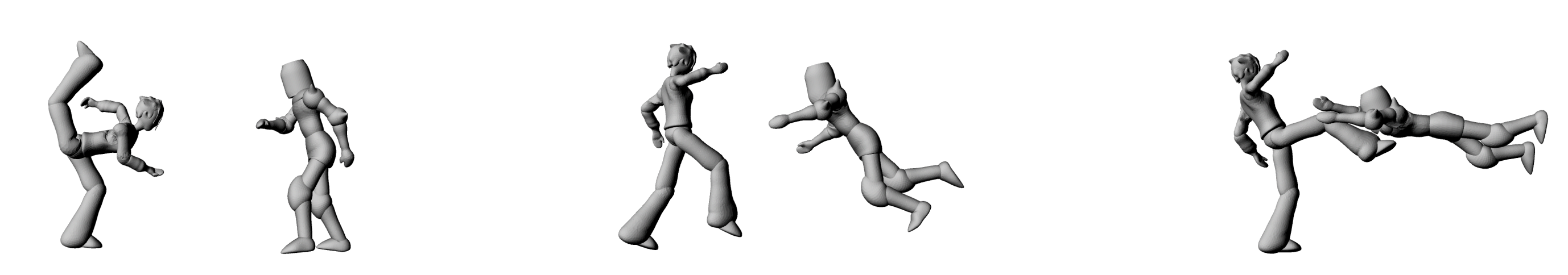

$$
\text { 新素 }
$$

\section{Problem}

Sketch as the most intuitive and powerful 2D design method has been used by artists for decades. However it is not fully integrated into current 3D animation pipeline as the difficulties of interpreting $2 \mathrm{D}$ line drawing into

Posing 3D characters from 2D input is a complex and open problem.

\section{Related Work}

Guay and colleagues use the line of action to pose the character with a single line to constrain the orientation of the skeleton structure.

The line of action: an intuitive interface for expressive character posing

$$
\text { - M. Guay, M. Cani, R. Ronfard }
$$

Hahn and colleagues proposed sketch abstractions to pose the character with sketches on the mesh for both the resting pose and the target pose.

- Sketch Abstractions for Character Posing - F. Hahn, F. Mutzel, S. Coros, B. Thomaszewski, M. Nitti, M. Gross, R. Sumner

\section{Our Approach}

We propose a new sketch based character posing system which is more flexible, efficient and it requires less input from the user. The character can be easily posed no matter the sketch represents a skeleton structure or shape contours.
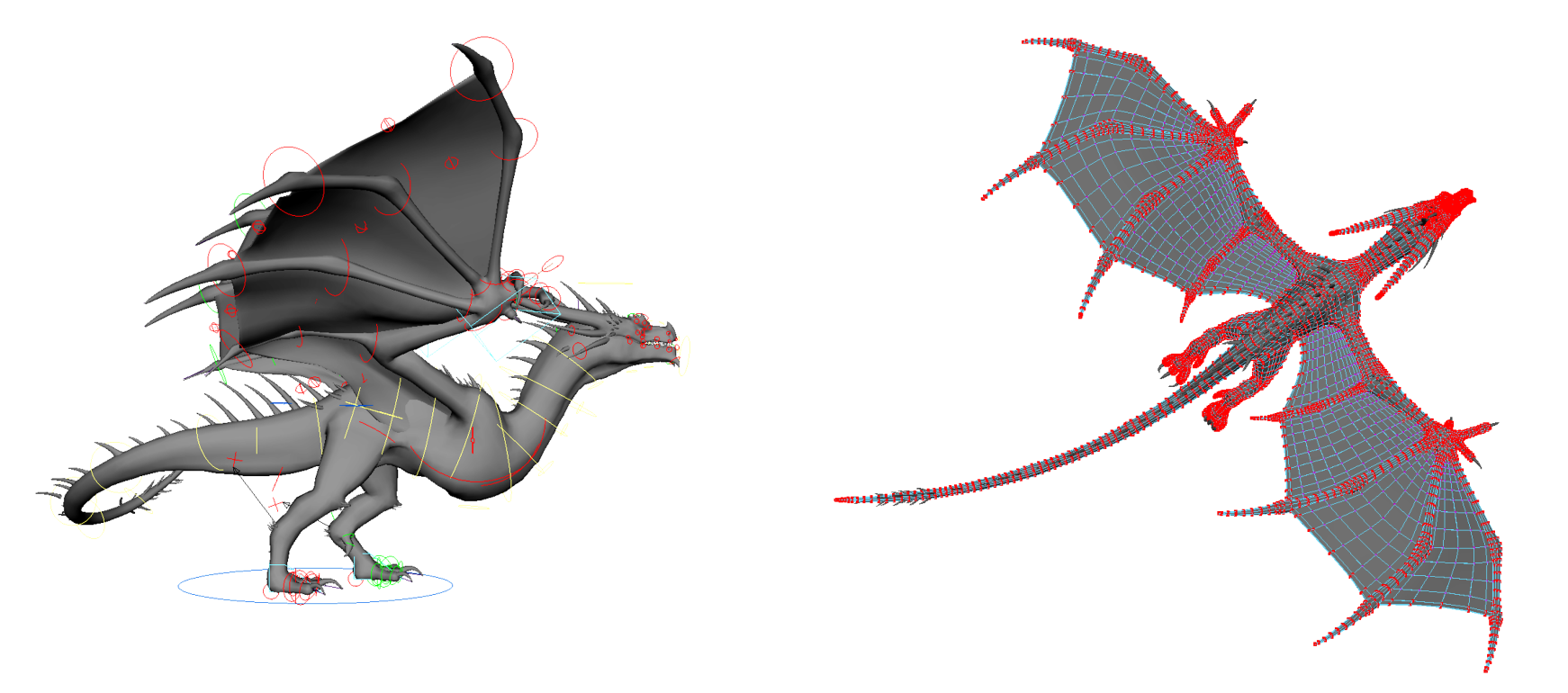

ut: Input:

- Character model $V$ and its rigging $p$. - Sampled sketch:

$$
Y=\left(y_{1}, y_{2}, \cdots, y_{M}\right)
$$

- Subset of vertices from the character mesh:

$$
V=\left(v_{1}, v_{2}, \cdots, v_{K}\right)
$$

including:

* the points are around the outlines;

the points are lying close to the projection
To match the two point sets $V$ and $Y$ and meanwhile deform $V$ to $Y$ as closely as possible (finding $p$ ) we formulate it as solving the following optimization problem:

$$
\min _{\omega, p} \sum_{i=1}^{M} \sum_{k=1}^{K} \omega_{k i}\left\|y_{i}-v_{k}(p)\right\|_{2}^{2}+\Phi(p)-\zeta \sum_{i=1}^{M} \sum_{k=1}^{K} \omega_{k i}
$$

such that:

$$
\begin{aligned}
& \text { - } \sum_{i=1}^{M+1} \omega_{k i}=1, k \in\{1,2, \cdots, K\} ; \\
& \text { - } \sum_{k=1}^{K+1} \omega_{k i}=1, i \in\{1,2, \cdots, M\} ; \\
& \text { - } \omega_{k i} \in\{0,1\} ;
\end{aligned}
$$

- $\omega=\left\{\omega_{k i}\right\}_{(K+1) \times(M+1)}$ is the correspondance matrix, where the last row and column are introduced to handle outliers;

- the second regularization term is used to add further constraints for searching candidate solutions in limited space;

- the third term is used to prevent treating too many points as outliers.

The objective function consists of:

- a linear discrete assignment problem for correspondence;

- a non-linear continuous problem for deformation
We can adopt an alternating strategy to solve the correspondence parameter $\omega$ and the rig parameter $p$ :

- By fixing $p$, we relax the binary $\omega$ to be a conlaxed subproblem by using Softassign and deterministic annealing.

By fixing $\omega$, the eney function can be solved

using a Newton-Raphson scheme.

\section{Results and Future work}

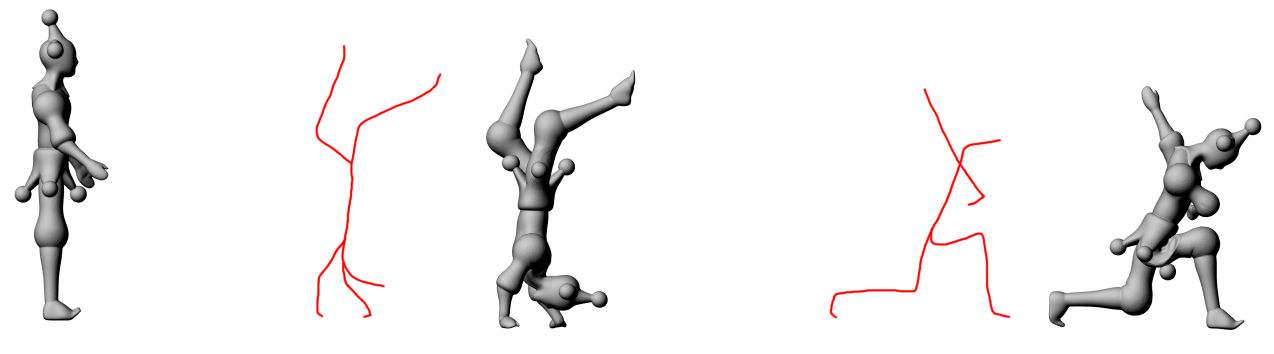

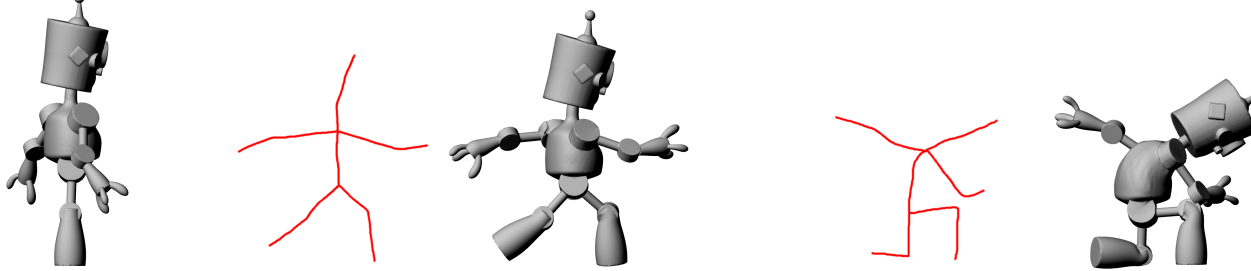

The current system solves the posing parameters to match the input sketch and the rigged mesh. We intend to incorporate shape deformation into our energy function in the future, offering a more complete tool for character animation. 